\title{
Application of a Three-Dimensional Reconstruction System in Breast Cancer with Ipsilateral Supraclavicular Lymph Node Metastasis: A Case Series
}

\author{
Piao Zhao Qiongyan Zou Liqin Yuan Lun Li Qitong Chen Dengjie Ouyang Wenjun Yi
}

Department of General Surgery, The Second Xiangya Hospital, Central South University, Changsha, China

\section{Established Facts}

- Existing imaging methods are not accurate enough to precisely judge the feasibility of supraclavicular lymph node dissection and the modes of ipsilateral supraclavicular lymph node metastasis.

Novel Insights

- Could the three-dimensional reconstruction system significantly benefit the precise assessment of the lesion area and facilitate supraclavicular lymph node dissection?

\section{Keywords}

Breast neoplasms · Breast cancer · Lymphatic metastasis .

Surgical therapy $3 \mathrm{D}$ reconstruction system .

Ipsilateral supraclavicular lymph node metastasis

\section{Summary}

Background: The role of supraclavicular lymph node dissection (SCLND) in breast cancer patients with ipsilateral supraclavicular lymph node metastasis (ISLM) remains controversial. So far, there have been no effective imaging methods to precisely judge the feasibility of SCLND and the modes of ISLM. Case Report: We innovatively applied a three-dimensional (3D) reconstruction system to assess the feasibility of SCLND preoperatively for 13 breast cancer patients with ISLM. Based on the 3D reconstruction system and intraoperative findings, we performed lymph node dissection of their lesion areas. Compared to computed tomography or ultrasonography, the 3D reconstruction system found more lymph nodes not only in the ipsilateral supraclavicular area but also in other areas in which metastasis may occur ( $p$ $<0.05$ ), and provided visual images pertaining to the re- lationship between the lymph nodes and major blood vessels, nerves, and muscles. Conclusion: The 3D reconstruction system could significantly benefit the precise assessment of the lesion area and facilitate subsequent relevant surgery.

(c) 2018 S. Karger AG, Basel

\section{Introduction}

Clinically, ipsilateral supraclavicular lymph node metastasis (ISLM) in breast cancer can be classified into 2 groups: synchronous and metachronous. The former is classified as stage IIIc (T1$4 \mathrm{~N} 3 \mathrm{M} 0$ ) disease, while there is a widespread impression that the latter is an incurable local recurrence.

For ISLM in breast cancer, individualized and multi-modality therapies, including adjuvant therapy, surgery (supraclavicular lymph node dissection (SCLND) and mastectomy), and radiotherapy, have been recommended [1]. Jung et al. [2] reviewed 111 breast cancer patients with pathologically proven ISLM and found that SCLND was not associated with additional survival benefits. In contrast, Chen et al. [3] showed that patients who received either excisional biopsy or total removal of the metastatic tumor

\section{KARGER}

() 2018 S. Karger AG, Basel 
Fig. 1. a Positron emission tomography/computed tomography image and $\mathbf{b} 3 \mathrm{D}$ reconstruction image of the 48-year-old female patient.
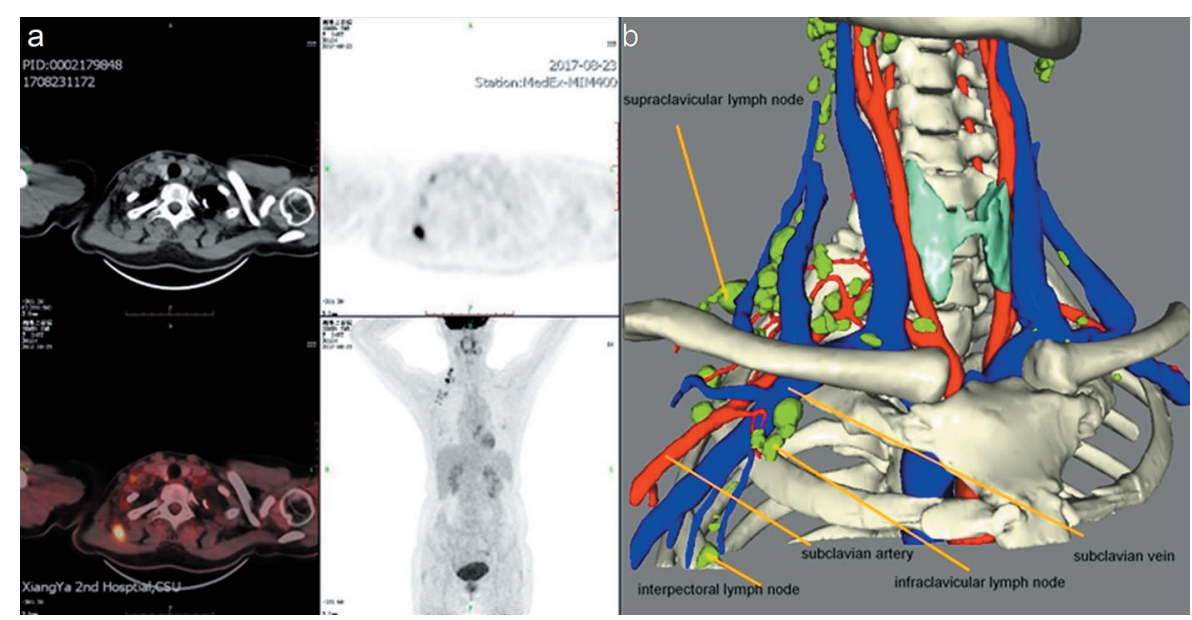

Fig. 2. a Computed tomography image and $\mathbf{b} 3 \mathrm{D}$ reconstruction image of the 45 -year-old female patient.

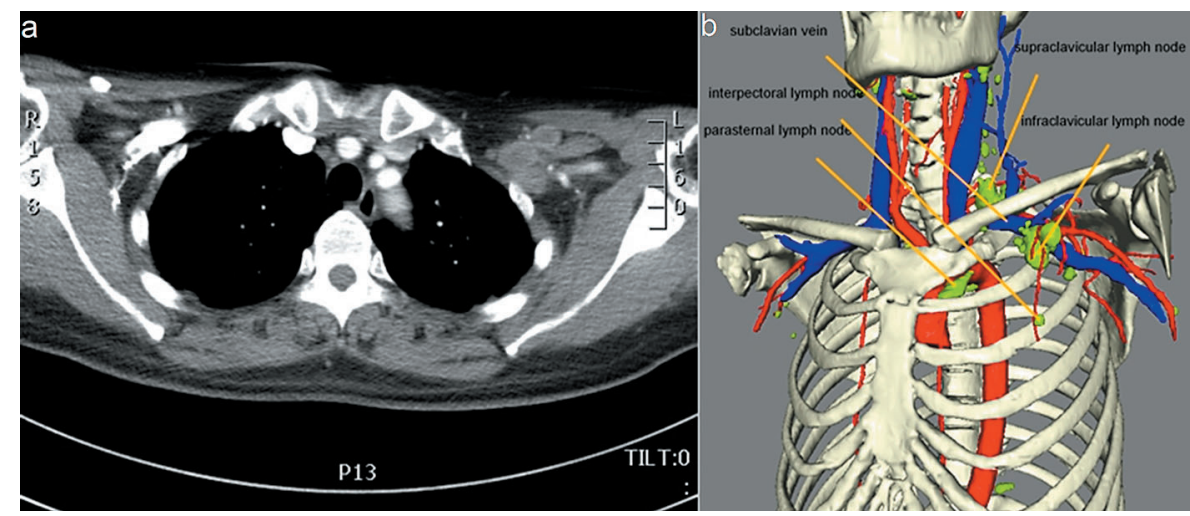

(dissection of neck level IV and V lymph nodes) had a better 5-year overall survival than those who underwent only fine-needle aspiration cytological analysis. However, Liu et al. [4] summarized typical complications of ISLM surgery, including lymphedema, hypoesthesia, abduction restriction of the shoulder joint, shape change of shoulder and upper arm abduction, facial edema, head and neck disorders, pleural effusion, and chylothorax. Because existing imaging methods like ultrasonography, computed tomography (CT), and positron emission tomography (PET)-CT are not accurate enough, the value and safety of SCLND remain debatable. Therefore, a precise method to judge the feasibility of SCLND and modes of ISLM is urgently needed.

Thus, we here report on a series of breast cancer patients with ISLM who were innovatively and effectively assessed using threedimensional (3D) reconstruction and subsequently underwent successful lymph node dissection.

\section{Case Report}

A 3D reconstruction system (INCOOL Medical Technology Co., Hangzhou, China) was applied in 13 breast cancer patients with ISLM at the Second Xiangya Hospital, Central South University, China between July 2017 and March 2018. The median age was 45 years (range 32-68 years). There were 6 cases of synchronous ISLM. 3 of them were reassessed after preoperative treatment and underwent mastectomy, axillary lymph nodes dissection, and SCLND. Pathologic complete response (pCR) was confirmed for all lymph nodes. The other 3 patients with synchronous ISLM did not receive modified radical mastectomy or mastectomy because the tumor could not be completely removed according to the $3 \mathrm{D}$ reconstruction assessment. The remaining 7 breast cancer patients had metachronous ISLM. 3 patients had previously received breast-conserving surgery and 4 had undergone modified radical mastectomy. All were evaluated using the 3D reconstruction system, and lymph node dissection of the lesion areas was subsequently performed. 1 patient underwent supraclavicular, infraclavicular, axillary, interpectoral, and parasternal lymph node dissection. Postoperative treatment was based on the molecular classification of the breast cancer tissues: 1 (7.7\%) patient was luminal A, 5 (23.1\%) patients were luminal B, 5 (23.1\%) were HER2-positive, and 2 (15.4\%) were triple-negative subtype.

There were 2 typical cases: A 48-year-old female breast cancer patient, 17 months after mastectomy and axillary lymph nodes dissection, was diagnosed with multiple ipsilateral lymph node metastases. The other case was a 45 -yearold female patient who received mastectomy and axillary lymph node dissection in September 2015, and ipsilateral axillary, supraclavicular, and infraclavicular lymph node metastases were found in August 2017. The 3D reconstruction system was applied to make a clear and direct display of the enlarged lymph nodes and their relationship with nerves and vessels (figs. 1, 2). Ultrasonography of the 2 patients provided no information. Axillary, and supraclavicular lymph node enlargement of the former patient and axillary, infraclavicular, and supraclavicular lymph node enlargement of the latter patient were detected by CT. The short axis diameters of the largest lymph nodes of the 2 patients measured with the $3 \mathrm{D}$ reconstruction system were 1.3 and $1.8 \mathrm{~cm}$, respectively. The smallest lymph node detected with the $3 \mathrm{D}$ reconstruction system was an interpectoral lymph node with a diameter of only $0.3 \mathrm{~cm}$. Based on the $3 \mathrm{D}$ reconstruction images and intraoperative findings, we successfully performed axillary, interpectoral, supraclavicular, and infraclavicular lymph node dissection for the former patient and axillary, interpectoral, supraclavicular, infraclavicular, and parasternal lymph node dissection for the latter patient; lymph node metastasis was pathologically confirmed. 
Fig. 3. Functional frameworks for the $3 \mathrm{D}$ recon-

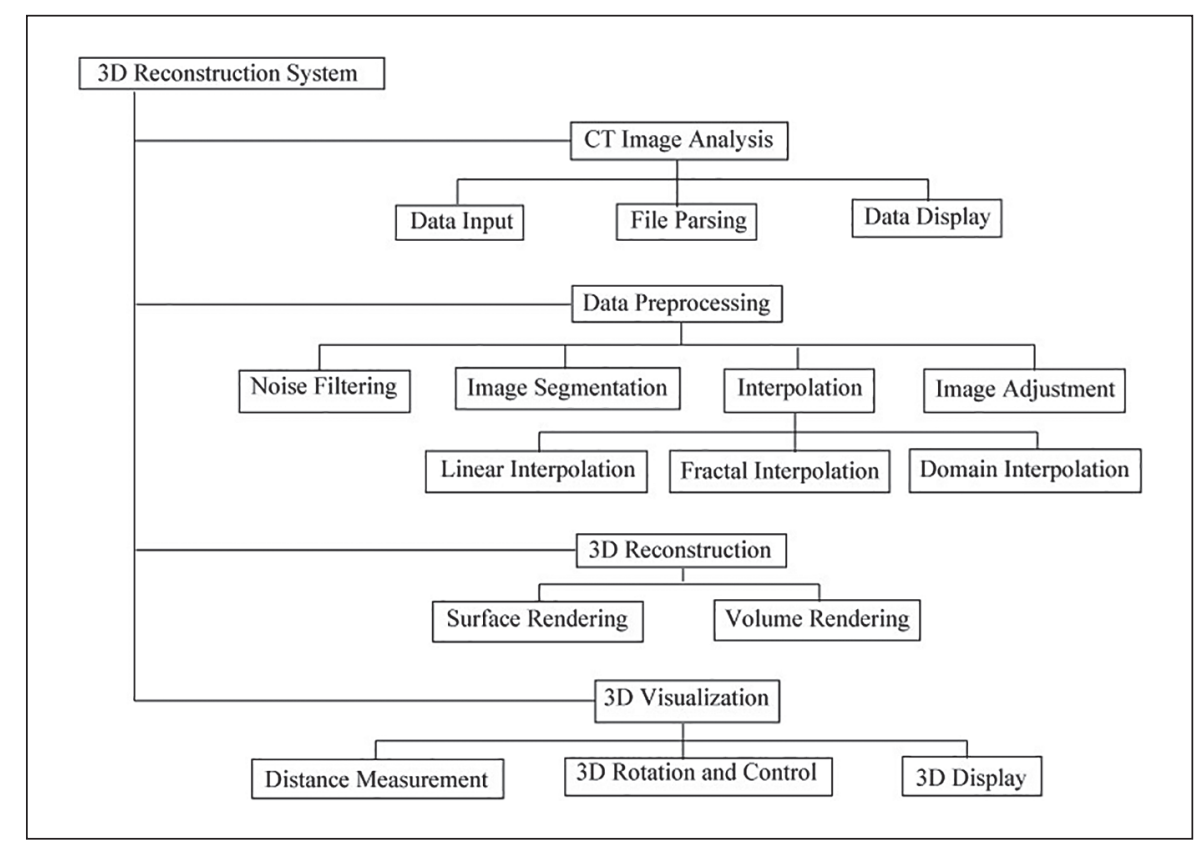
struction system.

Table 1. Suspected regions of lymph node metastasis according to each imaging method

\begin{tabular}{lll}
\hline Imaging method & Mean \pm standard deviation & p value \\
\hline 3D reconstruction system & $2.308 \pm 0.201$ & 1.000 \\
Ultrasonography & $1.077 \pm 0.201$ & 0.000 \\
Computed tomography & $1.385 \pm 0.201$ & 0.003 \\
\hline
\end{tabular}

To further explore the superiority of the 3D reconstruction system in detecting suspected lesion areas, we compared the difference between the 3D reconstruction system, ultrasonography, and CT in the 13 patients. The 3D reconstruction system was found to be more likely to detect the other 4 possible regions suspected to harbor metastasis besides the supraclavicular nodes $(\mathrm{p}<0.05)$, including axillary, infraclavicular, parasternal, and interpectoral lymph nodes (table 1). In short, compared to CT or ultrasonography, the 3D reconstruction system could provide visual images revealing the relationship between the lymph nodes and major blood vessels, nerves, and muscles, which benefited the preoperative assessment in terms of feasibility and security.

Our results indicate that the $3 \mathrm{D}$ reconstruction system could be used to make a feasibility assessment for completely removing the lesions and choose an appropriate surgical procedure the outcome of which may influence the prognosis. 3 cases of synchronous ISLM and 7 cases of metachronous ISLM received surgery after assessment with the $3 \mathrm{D}$ reconstruction system. In 3 cases of synchronous ISLM, the 3D reconstruction system did not report the suspected lymph nodes in the same way as ultrasonography or CT, and pCR of the lymph nodes was confirmed by pathology. Furthermore, the 3D reconstruction system benefited the assessment of multiple lymph node metastases besides the supraclavicular nodes, especially the interpectoral lymph nodes which were smaller in diameter (figs. 1,2). The 3D reconstruction system was clearly superior to CT and ultrasonography in the assessment of multiple lymph node metastasis (table 1).

Lymph node regions suspicious for metastasis according to each imaging method were analyzed using the matched $\mathrm{t}$-test. All statistical analyses were performed with the SPSS statistical package version 18.0 (IBM Corp., Armonk, NY, USA). Differences were considered statistically significant when $\mathrm{p}<0.05$.

\section{Discussion}

Good neck control in breast cancer patients with ISLM can achieve better survival, and surgery is one of the important multimodality treatments. Complications and variations in anatomy and the need to protect major blood vessels and nerves are tough challenges that need to be overcome by the surgeon. However, so far, there have been no effective imaging methods for precisely displaying the anatomic relationships between the enlarged lymph nodes and major blood vessels, nerves, and muscles.

The $3 \mathrm{D}$ reconstruction system used in this study is able to provide spatial visualization, manipulation, and measurement. In this system, the Digital Imaging and Communications in Medicine (DICOM) data from the CT image is read, preprocessed, and arranged exactly matching the real spatial positions of the voxels. Then, medical 3D images are created from the voxels. In terms of the processing workflow, this system consists of image preprocessing and input, $2 \mathrm{D}$ data preprocessing, and $3 \mathrm{D}$ rendering modules (fig. 3). It takes 5-6 h to finish the 3 steps and another $2-3 \mathrm{~h}$ to review for ordinary data; for complicated data, these steps take even more time.

The image preprocessing and input modules are able to read, store, and convert DICOM 3.0 standard data, mainly to provide support for a variety of medical image data formats. The CT images conform to the DICOM format, and their composition format and transmission methods are defined in detail in the DICOM standard. Utilizing this standard, an interface is established in the system through the $\mathrm{C}++$ language and the visualization toolkit to complete the input and output of the image data.

The $2 \mathrm{D}$ data preprocessing module is mainly used for data analysis and image segmentation, which form the basis for subsequent procedures. Before these steps, noise reduction through a median 
filter and useful parts of image extraction take place to prepare for subsequent $3 \mathrm{D}$ reconstruction.

Finally, the 3D rendering module uses surface rendering and volume rendering for reconstruction. Surface rendering can transform and rotate the image quickly and flexibly and is appropriate for drawing surface features of tissues and organs. Volume rendering directly processes all voxels in the $3 \mathrm{D}$ data field to synthesize the image with $3 \mathrm{D}$ effect; this has a good effect on the 3D display for tissues and organs with blurred shape features.

While we were able to show that our novel imaging modality better visualizes the supraclavicular lymph nodes, there were some limitations. Due to the short study time using a 3D reconstruction system, the number of patients in this case series was not large enough and follow-up data were insufficient. More work remains to be done to collect data and introduce the $3 \mathrm{D}$ reconstruction system to a larger patient collective.
In short, this system could provide valuable information for the preoperative assessment and guidance for the surgical procedure, display metastatic patterns, and discover small lesions difficult to detect by traditional imaging methods. This technique can enhance the visualization of the operative field more than any other previous imaging method and help surgeons to obtain a deeper comprehension of the spatial structure. We conclude that the $3 \mathrm{D}$ reconstruction system could significantly benefit the precise assessment of the lesion area and facilitate subsequent relevant surgery.

\section{Disclosure Statement}

The authors declare that they have no conflict of interest.

\section{References}

1 Fan Y, Xu BH, Liao YQ, Yao SY, Sun Y: A retrospective study of metachronous and synchronous ipsilateral supraclavicular lymph node metastases in breast cancer patients. Breast 2010;19:365-369.
2 Jung J, Kim SS, Ahn SD, et al: Treatment outcome of breast cancer with pathologically proven synchronous ipsilateral supraclavicular lymph node metastases. J Breast Cancer 2015;18:167-172.

3 Chen SC, Chang HK, Lin YC, et al: Prognosis of breast cancer after supraclavicular lymph node metastasis: not a distant metastasis. Ann Surg Oncol 2006;13: 1457-1465.
4 Liu XJ, Wang J, Li W: The complications and safety of supraclavicular lymph node dissection for invasive breast cancer with ipsilateral supraclavicular lymph node metastasis. Chin J Bases Clin General Surg 2016; 23:1040-1043. 